\title{
Presencia de síndrome de Burnout en una muestra de residentes y otorrinolaringólogos de Chile
}

\author{
Burnout syndrome in a sample of residents and otolaryngologists from Chile
}

Gonzalo Ortega $\mathrm{F}^{1}$, Giorgio Pio $\mathbf{R}^{1}$, Felipe Cardemil $\mathbf{M}^{1,2}$, Cristina Sade $\mathbf{B}^{3}$.

\begin{abstract}
RESUMEN
Introducción: El síndrome de Burnout o desgaste profesional relacionado con el trabajo se genera frente a un estrés laboral crónico en individuos que trabajan con personas. Se caracteriza por un agotamiento emocional, despersonalización y baja realización personal.

objetivo: Evaluar el síndrome de Burnout en los médicos residentes y otorrinolaringólogos (ORL) del país y establecer una relación entre las características sociodemográficas.

Material y método: Se realizó un estudio de corte transversal. Se envió el inventario Burnout de Maslach (MBI) mediante correo electrónico a todos los residentes y ORL miembros de la Sociedad Chilena de Otorrinolaringología, Medicina y Cirugía de Cabeza y Cuello (SOCHIORL). Se utilizó test exacto de Fisher para evaluar asociación entre variables cualitativas.

Resultados: De un total de 432 miembros de la SOCHIORL se recibieron 133 encuestas, de las cuales se analizaron 117 contestadas correctamente. El 100\% del total de los encuestados presentó Burnout ya sea de alto grado o intermedio. Los que presentaron mayor porcentaje de alto grado de Burnout son aquellos entre 25 y 35 años, con $53 \%$ ( $p$ $=0,03)$. El mayor grado de Burnout se observó en los residentes $(57 \%)(p=0,03)$.

Conclusión: El síndrome de Burnout tiene una alta prevalencia entre los ORL del país, especialmente en residentes y jóvenes ORL y quienes llevan pocos años en el desarrollo de la especialidad. Es necesario buscar herramientas para enfrentar y combatir esta realidad.

Palabras clave: Burnout, otorrinolaringología, Maslach.
\end{abstract}

\begin{abstract}
Introduction: Burnout syndrome is generated against a chronic work stress on individuals working with people. It is characterized by emotional exhaustion, depersonalization and reduced personal accomplishment.

Aim: To assess Burnout in residents and otolaryngologists (ORL) in the country and establish a relationship between sociodemographic characteristics.
\end{abstract}

1 Servicio de Otorrinolaringología Hospital San Juan de Dios - Universidad de Chile.

2 Departamento de Otorrinolaringología, Clínica Las Condes.

3 Departamento de Psiquiatría, Hospital Barros Luco Trudeau - Universidad de Chile.

Recibido el 3 de mayo de 2015. Aceptado el 15 de agosto de 2015. 
Material and method: A cross sectional study was conducted. Maslach Burnout Inventory (MBI) was sent via email to all residents and ORL members of the Chilean Society of Otolaryngology, Medicine and Head and Neck Surgery (SOCHIORL). Fisher exact test was used to assess the association between qualitative variables.

Results: Of a total of 432 members of SOCHIORL, 133 surveys were received, of which 117 were analysed. $100 \%$ of respondents had Burnout, either high or intermediate degree. Those with higher percentage of high Burnout are those between 25 and 35 years (53\%) ( $p$ $=0.03)$. The greatest degree of Burnout was observed in residents (57\%) $(p=0.03)$.

Conclusion: Burnout syndrome is highly prevalent among ORL in Chile, especially in residents and young ORL with few years in the medical practice of the specialty. It is necessary to search for tools to confront and combat this reality.

Key words: Burnout, Otolaryngology, Maslach.

\section{INTRODUCCIÓN}

El síndrome de Burnout, o desgaste profesional relacionado con el trabajo, fue descrito inicialmente en 1974 por Herbert Freudenberger ${ }^{1}$. Éste se produce frente a un estrés laboral crónico en quienes trabajan con personas, definiéndose como un agotamiento emocional, despersonalización y baja realización personal'. Modelos etiopatogénicos plantean que la génesis de este síndrome se basa en un balance entre factores internos (personalidad, necesidad de reconocimiento, entre otros) y factores ambientales (demanda del trabajo, ambiente laboral, responsabilidad en aumento, etc. $)^{2}$. Existen diversas estrategias para evaluar el síndrome de Burnout. Actualmente el Índice de Burnout de Maslach (Maslach Burnout Index - MBI) es el más utilizado, estando validado al español y para Chile ${ }^{3,4}$. Este consta de 25 preguntas divididas en los 3 ejes diagnósticos mencionados ${ }^{3,4}$. Se debe realizar el diagnóstico diferencial con depresión, síndrome de fatiga crónica y la alexitimia².

Estudios internacionales han evidenciado que este síndrome está fundamentalmente presente en cuidadores de la salud y otros tipos de trabajadores, pero el personal de enfermería es el que presenta la mayor cantidad de Burnout ${ }^{5,6}$. En relación a los médicos, se ha descrito que hasta $50 \%$ de ellos han presentado el síndrome de Burnout a lo largo de su carrera, siendo gran parte de éstos en el período de formación de médico o residente ${ }^{7,8}$. En términos de salud pública, el Burnout afecta la productividad de los funcionarios y trae como consecuencia mayores gastos en salud.
Es por ello que se han desarrollado diversas estrategias para prevenir e intentar solucionar este fenómeno ${ }^{7,9}$. En Chile se han realizado estudios de prevalencia de Burnout en servicios de pediatría, internos de medicina y otros, que se condicen con las prevalencias descritas en la literatura internacional ${ }^{10-12}$.

Actualmente en Chile no se han realizado estudios que evalúen la presencia del síndrome de Burnout en otorrinolaringólogos (ORL).

\section{OBJETIVO}

Medir la presencia del síndrome de Burnout en médicos residentes y ORL del país y evaluar la presencia de una asociación entre las características sociodemográficas.

\section{MATERIAL Y MÉTODO}

Se realizó un estudio de corte transversal durante el período julio-septiembre del año 2014. Se envió el inventario Burnout de Maslach (MBI) mediante correo electrónico a todos los residentes y ORL miembros de la Sociedad Chilena de Otorrinolaringología, Medicina y Cirugía de Cabeza y Cuello (SOCHIORL), utilizando la plataforma de encuestas de internet "www.surveymonkey. com". Se explicó a todos los contactados las características del estudio, y el carácter anónimo de la información personal entregada. Se utilizó test exacto de Fisher para evaluar asociación entre variables cualitativas. 


\section{RESULTADOS}

De un total de 432 miembros de la SOCHIORL se recibieron 133 encuestas, de las cuales se analizaron 117 contestadas correctamente. La muestra analizada corresponde al $27 \%$ de los ORL de nuestro país. De los encuestados, 74 fueron hombres $(63 \%)$ y 43 mujeres $(37 \%)$. Treinta y nueve encuestados (33\%) tuvieron entre 25 y 35 años de edad, 34 (29\%) entre 36 y 45 años, 17 (15\%) entre 46 y 55 años, 18 (15\%) entre 56 y 65 años, y sobre 65 años 9 sujetos (8\%).

Veinte y ocho encuestados (23\%) corresponden a residentes de ORL y $89(76 \%)$ a ORL propiamente tal. Del total, 80 sujetos $(68 \%)$ ejercían en la región metropolitana y 37 (32\%) en regiones. Del grupo de los otorrinolaringólogos, 36 (40\%) encuestados trabajaban tanto en el sector público como en el privado, 4 (5\%) exclusivamente en sector público, y 49 (55\%) sólo en sector privado.
El $100 \%$ del total de encuestados presentó Burnout ya sea en alto grado o intermedio. Cuarenta y siete $(40 \%)$ individuos presentan alto grado de Burnout y $70(60 \%)$ con grado intermedio. De los que trabajan exclusivamente en el sector público, $15(48 \%)$ presentan alto grado de Burnout, 18 $(36 \%)$ de los que sólo trabajan en sector privado y $14(38 \%)$ de los que trabajan en ambos sectores $(p=0,52)$. De los que trabajan en la región metropolitana (ver Figura 1), 36 (45\%) presentan alto grado de Burnout, en cambio, quienes trabajan en regiones sólo 30\% (11) ( $p=0,15)$.

En relación a los rangos etarios (ver Figura 2), Ios que presentan mayor porcentaje de alto grado de Burnout son aquellos entre 25 y 35 años, con $53 \%$, siendo esta diferencia estadísticamente significativa $(p=0,03)$. El mayor grado de Burnout se observó en los residentes (57\%), que desciende hasta llegar a $28 \%$ en el grupo con más de 10 años de egreso de la especialidad $(p=0,03)$ (ver Figura 3 ).

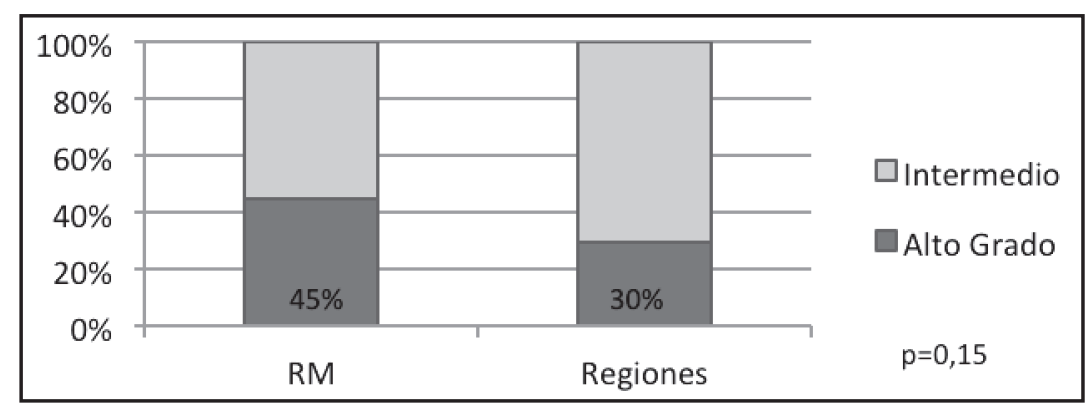

Figura 1. Distribución porcentual de otorrinolaringólogos que trabajan en la región metropolitana y en regiones y nivel de Burnout que presentan.

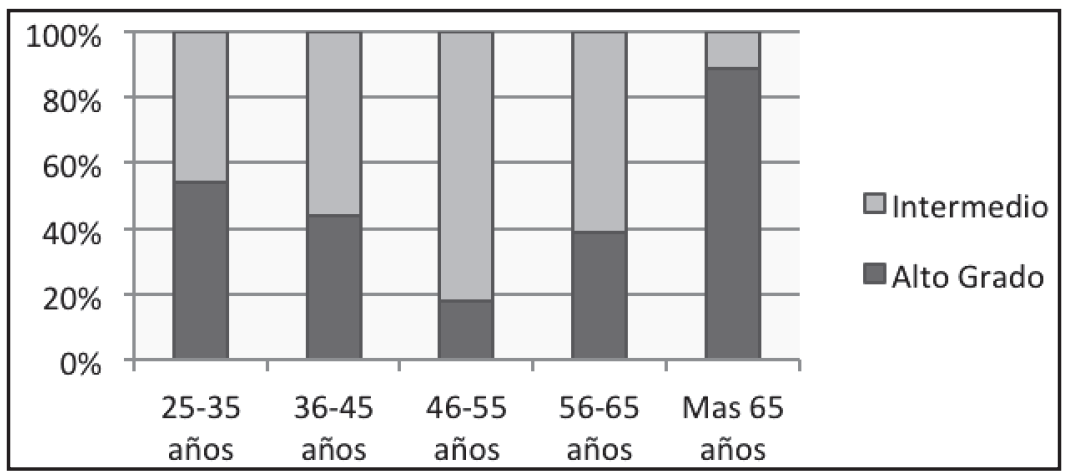

Figura 2. Distribución porcentual de los rangos etarios de los otorrinolaringólogos y niveles de Burnout que presentan. 


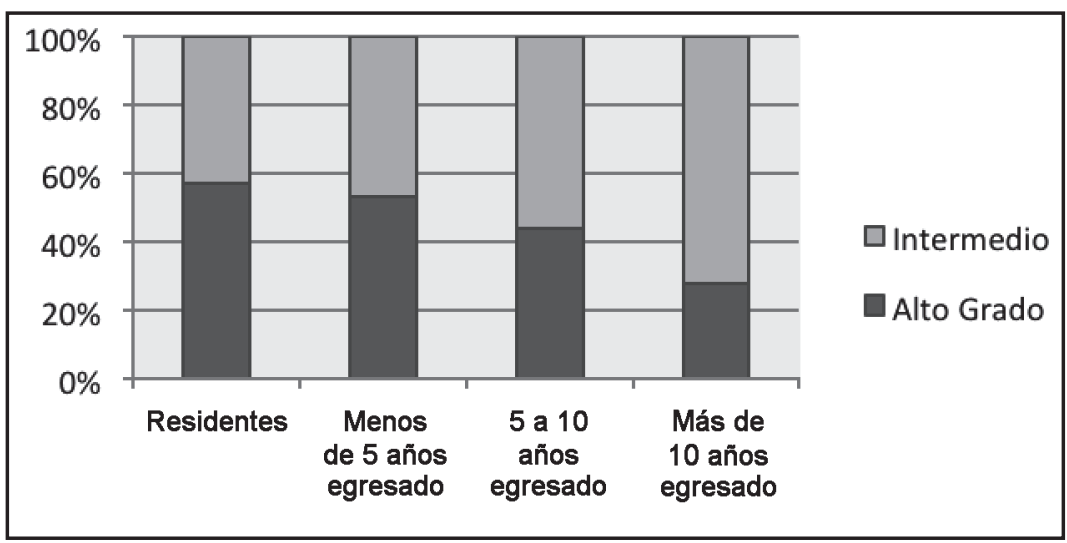

Figura 3. Distribución porcentual de los niveles de Burnout según si los médicos encuestados son residentes u ORL con distintos años de ejercicio de la especialidad.

\section{DISCUSIÓN}

Si bien el $100 \%$ de los encuestados presenta algún grado de Burnout según la encuesta de Maslach, se debe tener en consideración que solo se analizó al $27 \%$ de los ORL de la SOCHIORL, por lo cual se podría estar sobreestimando el grado de Burnout. Además se debe tener en consideración el momento en el cual fue respondida la encuesta, dado que sobrecargas emocionales o laborales podrían influir en las respuestas. Esto se puede evidenciar en la población ORL joven, la cual es la población de mayor riesgo, sobre todo los residentes de ORL. Esto es relevante dado que se ha descrito una asociación de enfermedades cardiológicas, musculoesqueléticas y alérgicas con el Burnout, como también estudios que plantean el síndrome como un factor de riesgo para desarrollar diabetes mellitus 2 y dislipidemia ${ }^{13,14}$.

Una revisión de Burnout en residentes entre los años 1983 a 2004 (15 estudios) demostró una prevalencia de Burnout que varía entre $17 \%$ y $76 \%$ según los años de residencia y la especialidad. Las especialidades quirúrgicas presentaban una prevalencia de $50 \%$, lo cual se aproxima al valor reportado por nuestros residentes de ORL ${ }^{15}$.

La Organización Mundial de la Salud y la Unión Europea han recalcado la importancia en la realización de terapias de prevención e intervención laborales para tratar el Burnout, dado los costos personales y sociales asociados ${ }^{16}$. Se han realizado diversos estudios en relación a cuáles son las mejores estrategias para prevenir el Burnout. Una estrategia es la terapia de aceptación y compromiso (Acceptance and commitment therapy). Esta es una terapia en base al comportamiento en la cual se enseña a evitar pensamientos estresantes o negativos, comportamiento que no permite resolver el problema de fond $0^{17}$. Otras estrategias apuntan al rediseño de tareas laborales, mejorar la supervisión, determinar objetivos laborales específicos para cada rol laboral, mejoras en las vías de comunicación, mejoras en el espacio físico laboral y flexibilidad laboral entre otras ${ }^{16}$.

Dado lo anterior, se debe evaluar la realización de distintas estrategias e intervenciones para disminuir y evitar el Burnout en los ORL del país. Estas deben estar a cargo de cada centro laboral y universitario.

\section{CONCLUSIÓN}

El síndrome de Burnout tiene una alta prevalencia entre los ORL del país, especialmente en residentes y jóvenes ORL y quienes llevan pocos años en el desarrollo de la especialidad. Es necesario buscar herramientas para enfrentar y combatir esta realidad. 


\section{BIBLIOGRAFÍA}

1. Gil-Monte PR, Peiro JM. Validez factorial del Maslach Burnout Inventory en una muestra multiocupacional. Psicothema 1999; 11(3): 679-89.

2. KaschKa WP, KorczaK D, Brolch K. Burnout: a fashionable diagnosis. Dtsch Arztebllnt 2011; 108(46): 781-7.

3. GIL-Monte PR. Factorial validity of the Spanish adaptation of the Maslach Burnout InventoryGeneral Survey. Salud Pública Mex 2002; 44(1): 33-40.

4. Manso-Pinto JF. Estructura Factorial del Maslach Burnout Inventory - Version Human Services Survey - en Chile. R Interam Psicol2006; 40(1): 115-8.

5. Finney et Al. Organizational stressors associated with job stress and burnout in correctional officers: a systematic review. BMC Public Health 2013; 13: 82.

6. Khamisa N, Peltzer K, Oldenburg B. Burnout in Relation to Specific Contributing Factors and Health Outcomes among Nurses: A Systematic Review. Int J Environ Res Public Health 2013; 10(6): 2214-40.

7. Romani M, Ashikar K. Burnout among physicians. Libyan J Med 2014; 9: 23556.

8. Dewa et Al. How does burnout affect physician productivity? A systematic literature review. BMC Health Services Research 2014; 14: 325.

9. Gilbody S, Cahill J, Barkham M, Richards D, Bee P, Glanville J. Can we improve the morale of staff working in psychiatric units? A systematic review. Journal of Mental Health 2006; 15(1): 7-17.
10. Ansoleaga E, Toro JP, God L. Malestar psicofisiológico en profesionales de la salud pública de la Región Metropolitana. Rev Med Chile 2011; 139: 1185-91.

11. Olmos-de-Aguilera R, Alfaro-Toloza P, Fuentealba M, Kirsten L. Presencia y correlación entre el síndrome de Burnout y empatía médica en internos de medicina de una escuela de regiones durante año 2011. Rev Soc Cient Estud Med Univ San Sebastián 2011; 6(1).

12. Ramírez A, Medeiro M, Muñoz C, Ramírez G. Alcances del síndrome de Burnout en estudiantes de medicina. Revista ANACEM 2012; 6(2): 110-2.

13. Melamed S, Shirom A, Toker S, Shapira I. Burnout and risk of type 2 diabetes: a prospective study of apparently healthy employed persons. Psychosom Med 2006; 68: 863-9.

14. Shirom A, Westman M, Shamal 0, Carel R. Effects of work overload and Burnout on cholesterol and triglycerides levels: the moderating effects of emotional reactivity among male and female employees. J Occup Health Psychol 1997; 2: 275-88.

15. ThomAs NK. Resident Burnout. JAMA 2004; 292(23): 2880-9.

16. Rodríguez R, Rivas $S$. Los procesos de estrés laboral y desgaste profesional (Burnout): diferenciación, actualización y líneas de intervención. Med Segur Trab (Internet) 2011; 57. Suplemento 1: 1-262.15.

17. Frögélı E, Djordjevic A, Rudman A, Livheim $F$, Gustavsson P. A randomized controlled pilot trial of Acceptance and Commitment Training (ACT) for preventing stress-related ill health among future nurses. Anxiety Stress Coping 2015 Mar 11:1-35. [Epubahead of print].

Dirección: Gonzalo Ortega F.

Servicio de Otorrinolaringología, Hospital San Juan de Dios

Huérfanos 3255, Santiago

E mail: gonzaloorteg@gmail.com 